\title{
KINETIC MODEL DEVELOPMENT ON SOURCE- SEPARATED ORGANIC WASTE FOR ETHANOL PRODUCTION BY S. cerevisiae STRAIN
}

\author{
Valeriy Bekmuradov ${ }^{1}$, Grace Luk $^{1}$, Michael Crupi ${ }^{2}$, Elsayed Elbeshbishy ${ }^{1}$ \\ ${ }^{1}$ Civil Engineering, Ryerson University, Canada \\ ${ }^{2}$ Optimum Waste \& Recycling Systems, Ltd, Canada
}

\begin{abstract}
Ethanol production from organic fraction of municipal solid waste with inclusion of construction/demolition waste can be an effective waste management strategy to overcome the growing problems with landfill space and dependency on conventional fuels. The main challenge in ethanol conversion is the high cost of processing in which pre-treatment, enzymatic hydrolysis and fermentation are the major steps. This study investigates impact of several key parameters, namely: $\mathrm{pH}$, temperature, adsorption capacity, cellulose hydrolysis rate, cell mass, enzyme and substrate loading doses on ethanol yield. The pre-treatment incorporates pre-processing and enzymatic hydrolysis steps through the use of a thermal screw press (TSP) and cellulose-organic-solvent based lignocellulosic fractionation (COSLIF) on the source-separated organic (SSO) waste to liberate fermentable sugars. Enzymatic hydrolysis experiments were featured with the addition of a commercially available enzyme complex, Accellerase 1500, to mediate the process and increase sugar yields. A kinetic model that uses a semi-mechanistic rate equation for cellulose hydrolysis was adapted and modified to accommodate batch simultaneous saccharification and co-fermentation (SSCF) process on pre-treated SSO waste by yeast, Saccharomyces cerevisiae DA2416. New experimentally defined SSO parameters have been fitted into a kinetic model to evaluate the sugar and ethanol yields. It was found that the model was capable of predicting ethanol productions with diminutive variance from experiments with substrate concentrations between $10 \mathrm{~g} / \mathrm{L}$ and $50 \mathrm{~g} / \mathrm{L}$. Model predictions from experimental data deviated significantly with substrate loading rate from 60 $\mathrm{g} / \mathrm{L}$ and higher. Fermentation results demonstrated that $S$. cerevisiae DA2416 produced ethanol in the range of $35-50 \mathrm{~g} / \mathrm{L}$, with ethanol yield of $0.48-0.50 \mathrm{~g}$ of ethanol/g sugar, in 5 days with $96 \%$ cellulose conversion. This study provides important insights for investigation on the use of SSO waste for ethanol production by S. cerevisiae DA2416. Furthermore, the model was proven to be a useful tool to facilitate future process optimization for up-scale bioreactors.
\end{abstract}

Keywords: Biomass, Organic Waste, Ethanol, Kinetic Modeling

\section{Introduction}

For many years, the main source of fuel for human society has come from fossil resources, which are not infinitive. Lignocellulosic biomass, on the other hand is a promising alternative to fossil fuels and it is the only foreseeable sustainable source of organic fuels and materials available to humanity (Shao, 2007). Lignocellulosic biomass such as the organic portion of solid waste is particularly attractive because of low cost and considerable availability. As estimated in (Ragauskas et al., 2006 and Zhang et al., 2006) it has a yearly supply of approximately 200 billion metric tons worldwide. However, the current cost of conversion creates a hold-up for commercial applications (Houghton et al., 2006). Among the strategies to reduce the processing costs are pre-treatment and usage of all fermentable sugars present in biomass with technologies available in today's market. Four approaches for cellulosic biomass processing featuring enzymatic hydrolysis have been reported: separate hydrolysis and fermentation (SHF), simultaneous saccharification and fermentation (SSF), simultaneous saccharification and co-fermentation (SSCF) and consolidating bioprocessing (CBP) (Shao, 2007). There are four biologically mediated events in each approach: cellulase production, cellulose hydrolysis, pentose and hexose fermentations (Department of Energy, USA, 1998,Lynd, et al., 2002). 
SHF and SSF approaches are featured in many experimental designs for immediate implementation, while SSCF and CBP reqiure more research from deep-rooted process development (Shao, 2007). It has been reported (Department of Energy, USA, 1998) that a major disadvantage of SHF was inhibition of cellulose hydrolysis by glucose. They were unable to obtain glucose concentrations higher than 5.5\% using SHF. The SSF approach, featuring enzymatic hydrolysis and fermentation of hexose in one integrated step, considerably increases inhibition to cellulase by cellulose hydrolysis products (Zhang et al., 2009). The SSCF process is similar to SSF except that hexose and pentose fermentations occur in one step. Unlike SHF and SSF, the SSCF process offers potential for more streamlined processing and a lower capital cost (Lynd et al., 2002; Xiao et al., 2004).

The SSCF approach was chosen in this work. SSCF has become more attractive with the emergence of new microorganisms that produce ethanol at a high yield from both glucose and xylose and reduce inhibition hydrolysis by xylose (Kim and Lee, 2005).

The CBP approach has a similar prospective but requires a higher temperatute for the enzmatic hydrolysis reaction than SSCF. Moreover, CBP uses native Clostridium thermocellum which produces ethanol with significant amounts of acetic acid and cannot utilize xylose (Wyman, 1999).

Normally the process for ethanol production from lignocellulosic biomass is initiated with physiochemical pretreatment to increase the exposure of substrate to enzymatic hydrolysis followed by the biological conversion of resulting sugars to ethanol by a chosen fermenting strain. A recombinant strain of S. cerevisiae DA2416, which is capable of fermenting both glucose and xylose to produce ethanol at high yield, was used in this work. The $S$. cerevisiae DA2416 strain has by-passed problems with glucose repression, by taking advantage of xylose utilization pathways. As a result, key fermentation parameters (ethanol yield and inhibition) can be improved further for greater results in the fermentation phase on SSO waste.

Lignocellulosic biomass, such as pre-processed SSO waste, was used in this work as a feedstock for all enzymatic hydrolysis and fermentation processes. Due to its compositional analysis and relatively easy pretreatment, SSO was chosen as a potential substrate for future industrial applications.

An existing kinetic model was adopted from (Zhang, 2008) and modified in this work to predict batch SSCF on SSO waste by glucose and xylose utilizing strain S. cerevisiae DA2416. This model accounts for cellulose and hemicellulose enzymatic hydrolysis and competitive uptake of glucose and xylose. There are only a few published studies on the conversion of cellulose and hemicellulose via SSCF (McMillan et al., 1999; Teixeira et al., 2000; Kim and Lee, 2005) and only few kinetic models have been proposed in the literature (Shao, 2007, Zhang, 2008). A kinetic model development used in this work was based on a semi-mechanic rate equation for cellulose hydrolysis as initially proposed by (South et al., 1995) and further modified by (Shao, 2007) and (Zhang, 2008) to accommodate cellulose and hemicellulose hydrolysis. The parameters presented in (Shao, 2007) and (Zhang, 2008) were based on data for paper sludge only. Therefore, new values of interest such as adsorption capacity, enzymatic hydrolysis constant, ethanol inhibition and ethanol yield were recalculated to accommodate batch mode SSCF, particularly on SSO waste.

The overall goal of this work is to better comprehend the process of converting SSO waste via SSCF approach to produce ethanol. Specific objectives include: 1) revise batch SSCF, specifically for the SSO waste by means of adaptation and further amendment of an existing kinetic model; 2) examine the impact of major variables involved in the performance of SSCF on SSO waste (temperature, pH, adsorption capacity, enzymatic hydrolysis rate constant, ethanol inhibition and ethanol yield); 3) experimentally obtain new parameters for a tailored kinetic model to predict batch SSCF on SSO waste and compare results of model prediction and experiments. 


\section{Materials and Methods}

The $S$. cerevisiae DA2416 recombinant strain used in this study was kindly provided by Dr. Yong-Su Jin from the Department of Food Science and Human Nutrition, University of Illinois, USA. It was kept at $-80^{\circ} \mathrm{C}$ in $30 \%$ (v/v) glycerol for storage. The enzyme complex Accellerase 1500 used in the hydrolysis experiments was a gift of Sigma Aldrich Corp., USA.

The SSO waste utilized in this work was initially pre-processed mechanically, under high temperature $\left(120^{\circ} \mathrm{C}\right)$ and pressure (over 50 bars) with a thermal screw press to form a dry stable mass. SSO samples were prepared as a heterogeneous substrate by blending demolished construction waste, approximately $20 \%$ in form of woodchips and organic green bin waste as in (Bekmuradov et al., 2014a). Optimum Waste Recycling Systems, Toronto, Canada, supplied the biomass feedstock used in this work (Optimum waste and Recycling System, 2010). Prior to testing, the SSO waste was oven dried at $45^{\circ} \mathrm{C}-50^{\circ} \mathrm{C}$ for 48 hours.

The COSLIF method, which uses cellulose solvent (phosphoric acid) and organic-solvent (ethanol), was applied to the SSO waste followed by enzymatic hydrolysis and fermentation processes (Zhang et al., 2007; Sathitsuksanoh et al., 2009; Rollin et al., 2011). Five grams of dry lignocellulose was placed in a $250 \mathrm{~mL}$ centrifuge bottle and then mixed with $40 \mathrm{~mL}$ of $85 \%$ concentrated phosphoric acid using a glass rod. The solid/liquid slurry was placed in a bench-top shaking incubator at $150 \mathrm{rpm}$ and $50^{\circ} \mathrm{C} \pm 0.2^{\circ} \mathrm{C}$ for 2 hours. One hundred $\mathrm{mL}$ of ethanol was then added and mixed well. After centrifugation at 7000rpm at room temperature for 15 minutes, the supernatant was decanted. The solid pellet was then re-suspended with $200 \mathrm{~mL}$ of ethanol and centrifuged. The supernatant again was decanted. Next, the solid pellet was re-suspended with $200 \mathrm{~mL}$ of distilled water and centrifuged two times and stored in a freezer for a short period of time.

Enzymatic hydrolysis experiments were carried out with the addition of commercially available enzyme, Accellerase 1500. After thawing, the treated solid pellet containing amorphous cellulose was neutralized to $\mathrm{pH}$ 4.8-5.0 by $\mathrm{NH}_{4} \mathrm{OH}$. The SSO samples were then brought to $50^{\circ} \mathrm{C}$ before adding $30 \mathrm{FPU} / \mathrm{g}$ glucan of Accellerase 1500. Both the $\mathrm{pH}$ value and temperature described were the optimum conditions for the Accellerase 1500 enzyme to mediate hydrolysis and release as many fermentable sugars as possible (Dowe and McMillan, 2008). The hydrolysis experiment was conducted in the shaking incubator (MAXQ4450). The incubator was set at 250rpm to keep solids in constant suspension with the temperature of $50^{\circ} \mathrm{C}$ for 72 hours. Samples were taken and measured for sugar content at specified times: 0, 12, 24, 48 and 72 hours. The relevant composition of the SSO was the same as reported in (Bekmuradov et al., 2014b).

Protein content of the SSO substrate was measured by Lowry modified method (Thermo Fisher Scientific Inc., 2011). Adsorption of cellulases onto SSO substrate was done by mixing them in an incubator shaker at $100 \mathrm{rpm}$ in an Innova-40 shaker, at a temperature of $25^{\circ} \mathrm{C}$, in $10 \mathrm{~mL}$ glass tubes, under controlled $\mathrm{pH}$ and concentration of cellulases. Centrifugation of the whole reaction tube followed an incubation period. Then unbound cellulases present in the supernatant were decanted off. The amount of cellulase adsorbed onto a solid substrate was determined as the difference between the total amount of cellulase initially applied [Einit] and the amount of free cellulase in the solution [Enon ads]. The amount of free cellulases in the solution was measured by "in situ" and rapid UV spectrophotometer technique (Liu et al., 2011; Wang et al., 2012). The technique determines free cellulase concentration in the solid SSO substrate suspension from the second derivative of the absorption spectra at $750 \mathrm{~nm}$ with respect to wavelength through calibration. Each data point in the plots was an average of 5 replicates.

The carbohydrate content of SSO was determined via quantitative saccharification (QS) method based on 2 hours incubation in $72 \mathrm{wt} \% \mathrm{H}_{2} \mathrm{SO}_{4}$ at $30^{\circ} \mathrm{C}$ (Ruiz and Ehrman, 1996;McMillan et al., 1999; Moxley and Zhang, 2007). The cell mass was determined by counting colony forming units on agar plates as described in (Zhang et al., 2009). 
Following enzymatic hydrolysis, batch soluble sugar fermentation was carried out to evaluate ethanol yields from SSO samples as a result of conversion using recombinant strain - S. cerevisiae DA2416. Soluble sugar batch fermentation was performed in $250 \mathrm{~mL}$ serum bottles with $100 \mathrm{~mL}$ working volume. Temperature was maintained at $30^{\circ} \mathrm{C}$ and $\mathrm{pH}$ was controlled at 6.0 by $1 \mathrm{M}$ potassium hydroxide $(\mathrm{KOH})$ as suggested by a previous study (Mohageghi et al., 2004). Compositional analysis of the samples for ethanol concentrations was carried out at $0,12,24$ and 48 hours by high performance liquid chromatography (HPLC).

The kinetic model adapted in this study uses a semi-mechanistic rate equation for cellulose hydrolysis as proposed by (South et al., 1995) and further modified as in (Zhang et al., 2009). The parameters presented in Zhang's kinetic model were based on cellulose and hemicellulose hydrolysis for pre-treated paper sludge. In this study adsorption parameters were recalculated based on overall carbohydrate content of pre-treated SSO waste. The binding capacity or specific capacity of the carbohydrate component for cellulase of SSO samples was obtained using Langmuir isotherms. The cellulose hydrolysis rate constant was in the range of 0.662 to 0.725. The remaining cellulose hydrolysis parameters were as reported by (Zhang, 2008). Experimental data on glucose and xylose consumptions and growth parameters were fitted using the non-linear function of Polymath 5.1 (Polymath Software). All other parameters were dynamically fitted with the curve fitting function in the Berkeley Madonna computer program with a fourth-order Runge-Kutta algorithm. Runs were performed on a standard laptop.

A sensitivity analysis was carried out based on the least square method (Stigler, 1986; Bretscher, 1995). It was performed to test the impact of the value of several important constants on the model prediction of ethanol production if the constant had changed to a value $\pm 10 \%$ from its experimentally measured value. The analysis was performed to determine the difference between experimental data and modified kinetic model predictive ability.

\section{Results and Discussion}

Due to its prospect for commercial application, SSO waste was chosen as the substrate to evaluate the values on sugar and ethanol yields by fermentation using S. cerevisiae DA2416 strain.

The SSO composition was previously analyzed (Mirzajani, 2009; Ehsanipour, 2010; Bekmuradov et al., 2014a) and is shown in Table 1:

Table 1 Composition of the SSO on dry weight.

\begin{tabular}{ll}
\hline Glucose & Percentage \\
\hline Xylose & $31 \%$ \\
\hline Other sugars & $19 \%$ \\
\hline Extractives & $7 \%$ \\
\hline Lignin & $16 \%$ \\
\hline Total & $27 \%$ \\
\hline
\end{tabular}

Approximately, more than half of the original sample was composed of moisture. Essential polymeric sugars in the oven dried SSO samples included: $31 \%$ glucose, $19 \%$ xylose, $7 \%$ of other sugars, $16 \%$ extractives and $27 \%$ of lignin (Mirzajani, 2009). These homogeneous samples had a $\mathrm{pH}$ range of 5.2-5.5 and consisted of around $80 \%$ food waste and approximately $20 \%$ wood chips (Douglas fir type). The SSO samples pre-treated by concentrated phosphoric acid $(85 \% \mathrm{w} / \mathrm{w})$ and ethanol $(95 \% \mathrm{v} / \mathrm{v})$ were hydrolyzed and glucan digestibility was 
found to be $72 \%$ after 24 hours and $90 \%$ after 72 hours. The high glucan digestibility was achieved for the COSLIF-pre-treated SSO with addition of 30 FPU/g glucan of Accellerase 1500 (Bekmuradov et al., 2014b).

Recognizing that cellulase mixtures contain a mixture of cellulase and hemicellulase portions which bind to cellulose and hemicellulose, adsorption capacity constant " $\sigma$ " was recalculated using the modified method of Lowry and resultantly were in the range of 0.264 to 0.280 . Similarly, the binding capacity CS was determined as between $0.442 \mathrm{~g}$ protein/g carbohydrate and $0.466 \mathrm{~g}$ protein/g carbohydrate using Langmuir isotherms. And cellulose hydrolysis rate constant $-\mathrm{k}(1 / \mathrm{h})$ had a range values of 0.662 and 0.725 . The remaining cellulose enzymatic hydrolysis parameters were adapted (Zhang, 2008). Adsorption of Accellerase 1500 cellulase to the SSO waste samples was evaluated after hydrolysis was allowed to proceed for specified time, $(6,12,24,36,48$, 60 and 72 hours) resulting in various values for fractional conversion up to $85 \%$ as shown in Figure 1.

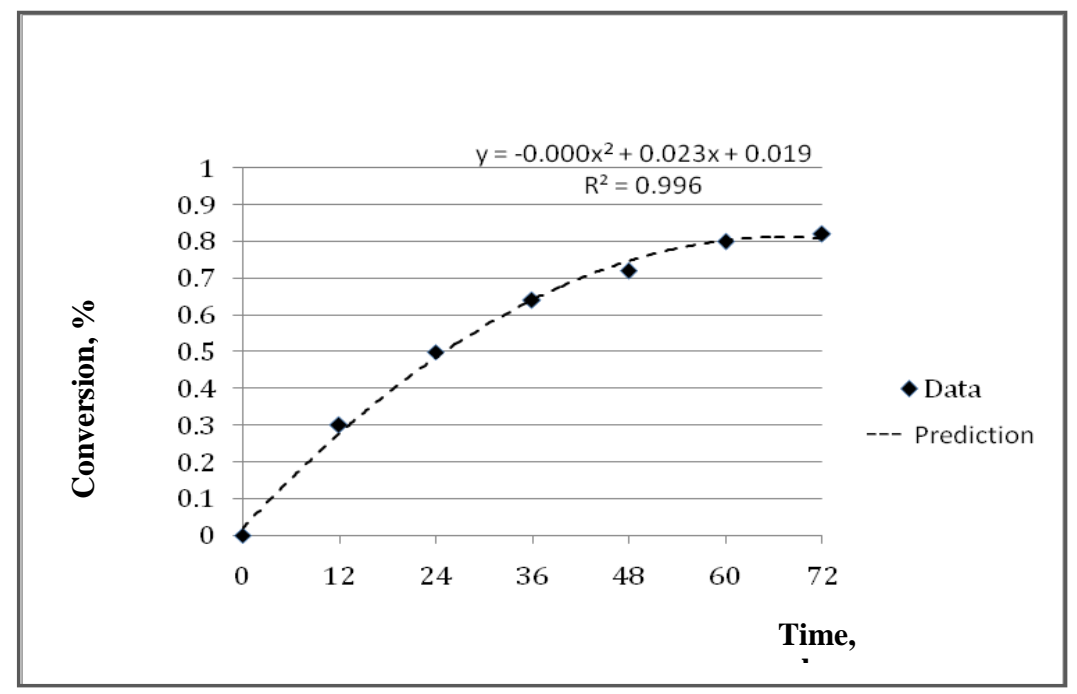

Figure 1: Conversion data for pre-treated SSO samples using Berkeley Madonna software

The predictions were confirmed by experimental data obtained during the addition of Accellerase 1500 enzyme complex to SSO samples throughout the course of hydrolysis. The predictions over the time of reaction are almost identical. The good fit obtained in this study suggests that for the SSO samples it seems reasonable to assume a constant adsorption capacity normalized to the amount of cellulose remaining and there is no reason to hypothesize adsorption affinity as a function of a conversion.

Adsorption parameters KS and $\sigma \mathrm{S}$ (Table 2) were then fit to the data of all conversions by minimizing the sum of squares for the predicted and observed data. The new adsorption parameters with conversion data were used to fit the parameters k, c and e in the cellulose rate equation of (South et al., 1995). The values of parameters are presented in Table 2:

Table 2 Parameter values for SSCF of the SSO

\begin{tabular}{lll}
\hline $\mathrm{K}_{\mathrm{S}}$ & $0,442-0,466$ & This work \\
\hline$\sigma_{\mathrm{S}}$ & $0,264-0,280$ & This work \\
\hline $\mathrm{K}$ & $0,662-0,725$ & This work \\
\hline $\mathrm{E}$ & $0,510-0,516$ & This work \\
\hline $\mathrm{C}$ & 0 & Shao, 2007
\end{tabular}


With parameter values in hand for adsorption, hydrolysis and fermentation, a pre-existing kinetic model for SSCF was chosen and modified to account a newly defined SSO feedstock constant. In view of this, we selected a simple correlation model reported in the following equation (1) from (Zhang, 2008):

$$
r_{X n}=\frac{X I}{G I} \times r_{G n} \quad \text { Equation (1) }
$$

where: $r_{G n}$ and $r_{X n}$ - formation of glucan and xylan respectively; $G I$ and $X I$ - initial glucan and xylan concentrations.

Equation (1) above describes the correlation of glucan and xylan hydrolysis and is derived from the simple relationship of $\mathrm{X} 1=\mathrm{X} 2$ in which $\mathrm{X} 1$ and $\mathrm{X} 2$ are the conversion of glucan and xylan respectively. To understand the enzyme hydrolysis performance with the $S$. cerevisiae DA2416 strain, the percentage of glucan and xylan conversion was calculated. The average glucan conversion to monomer sugars was $96 \%$, and the average xylan conversion to monomer sugars was $94 \%$ at $30^{\circ} \mathrm{C}$. However, we observed a slightly higher residual xylose accumulation than residual accumulation of glucose during experimental tests. The slower consumption rate of xylose than glucose was consistent with the fermentation of soluble sugars in other studies as well utilizing $S$. cerevisiae (Kuyper et al., 2005; Zhang and Lynd, 2010, Thermo Fisher Scientific Inc., 2011).

Before SSCF runs, Accellerase 1500 enzyme were added and mixed with yeast inoculum into a vessel at optimum value of 30FPU to increase the sugar's accessibility to cells during the mass transfer limited period. As a result, high glucan digestibility $(>90 \%)$ was achieved. Batch soluble sugar fermentation experiments were carried out to find the fermentation related constants, exclusively ethanol inhibition and yield in SSCF kinetic model by performance of recombinant strain - S. cerevisiae DA2416. In S. cerevisiae strains, there are a large number of genes encoding hexose transporters (Reifenberger et al., 1997), which are also believed to be function with low affinity xylose transporters in recombinant xylose utilizing S. cerevisiae (Sedlak and Ho, 2004). Based on this examination, a competitive substrate inhibition model for growth in glucose and xylose by S. cerevisiae DA2416 was chosen to capture the growth kinetics. Inhibition of growth and fermentation has been described using different equations in the literature, including exponential inhibition, linear inhibition, and linear inhibition beyond threshold (van Uden, 1989). Among them, a threshold linear inhibition model was chosen because it fit best with the data. A threshold linear inhibition model equation (2) as described elsewhere (South et al., 1995) accounts for glucose fermentation with an additional term representing sugar uptake from xylose and inhibition from ethanol:

$$
\mu_{G l}=\left[\frac{X \times \mu_{G l}^{M a x} \times G_{l}}{\left(K_{G l}+G l+I_{1} \times X_{l}\right)}\right] \times\left(1-\frac{E t h}{E t h_{G l}^{M a x}}\right) f^{1} \quad \text { Equation (2) }
$$

where: $\mu_{G l}^{M a x}$ and $E t h_{G l}^{M a x}$ - maximum specific growth rate and

maximum ethanol concentration for growth on glucose respectively;

$G_{l}, X_{l}, E t h$ - concentration of glucose, xylose and ethanol respectively;

$K_{G l}, I_{1}, f^{1}$ - related constants.

The rate of formation of xylose was described by an approach similar to that used for glucose formation shown in equation (3) from (South et al., 1995):

$$
\mu_{X l}=\left[\frac{X \times \mu_{X l}^{M a x} \times(X l-X l T)}{K_{X l} \times X+X l+I_{2} \times G l}\right] \times\left(1-\frac{E t h}{E t h_{X l}^{M a x}}\right) \quad \text { Equation (3) }
$$

where: $\mu_{X l}^{\operatorname{Max}}$ and $E t h_{X l}^{M a x}$ - maximum specific growth rate and maximum ethanol concentration for growth on xylose respectively;

$$
K_{X l}, I_{2} \text { - related constants; }
$$




\section{$X l T$ - threshold concentration.}

Values for inhibition factors I1 and I2 in this work were found to be 0.108 and 6.032 respectively, indicating that the inhibition of xylose utilization by glucose is more than 50 times stronger than the inhibition of glucose utilization by xylose. In order to test the SSCF performance on SSO feedstock with newly redefined constants, batch fermentations were carried out in a separate series of experimental evaluation at different initial substrate concentrations of $10 \mathrm{~g} / \mathrm{L}, 50 \mathrm{~g} / \mathrm{L}, 60 \mathrm{~g} / \mathrm{L}$ and $100 \mathrm{~g} / \mathrm{L}$ with the enzyme loading of $30 \mathrm{FPU}$ cellulase. The model accurately predicts the sugar and ethanol concentration along with cell mass concentration for substrate concentration ranging from $10 \mathrm{~g} / \mathrm{L}$ and $50 \mathrm{~g} / \mathrm{L}$ (Figures 2-3), but not for the $60 \mathrm{~g} / \mathrm{L}$ and higher.

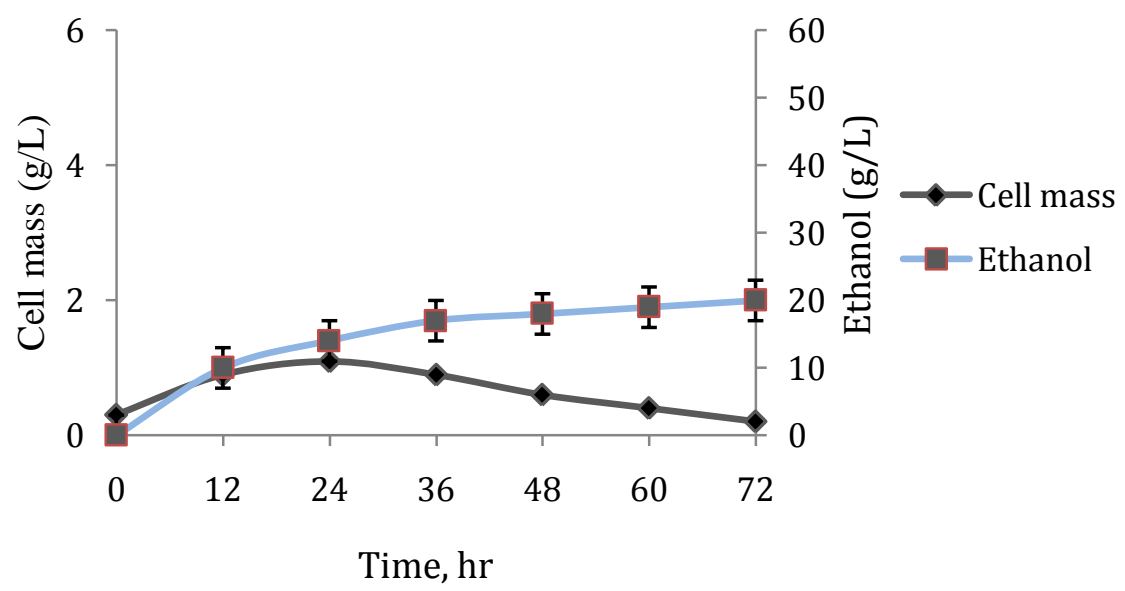

-Cell mass-exp—Cell mass-model= Ethanol-exp— Ethanol-model

Figure 2: Experimental data and kinetic model prediction for SSO samples (substrate concentration $10 \mathrm{~g} / \mathrm{L}$ ) 


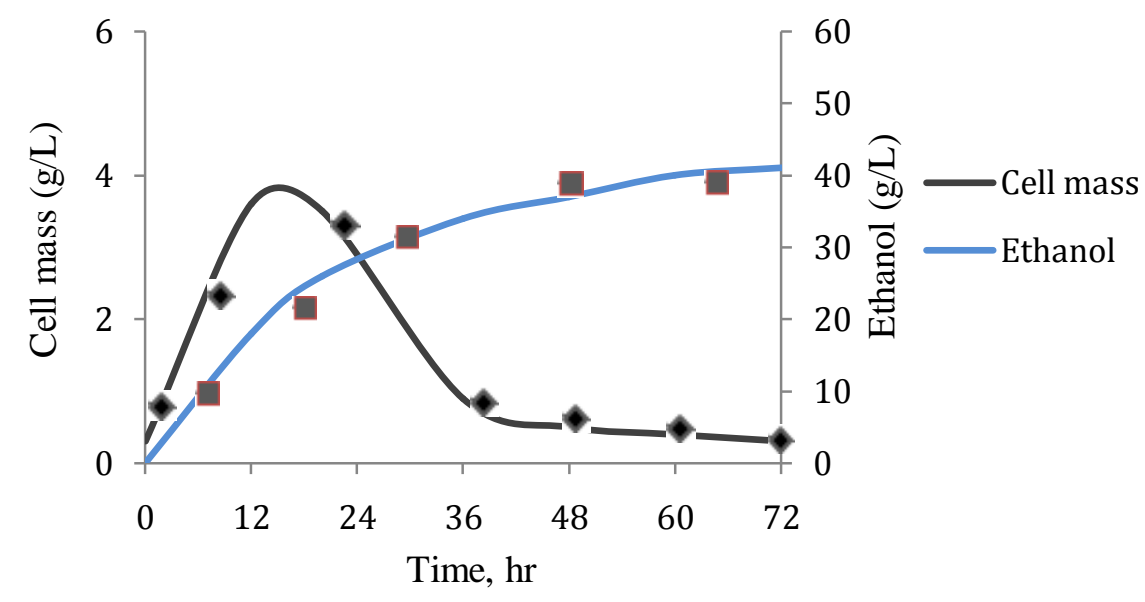

-Cell mass-exp—Cell mass-model ${ }^{\mathbf{1}}$ Ethanol-exp—Ethanol-model

Figure 3: Experimental data and kinetic model prediction for SSO samples (substrate concentration 50g/L)

In higher substrate concentration mode at $60 \mathrm{~g} / \mathrm{L}$, reaction was deviated from experimental values as shown in Figure 4 suggesting that ethanol inhibition is not a factor causing this discrepancy.

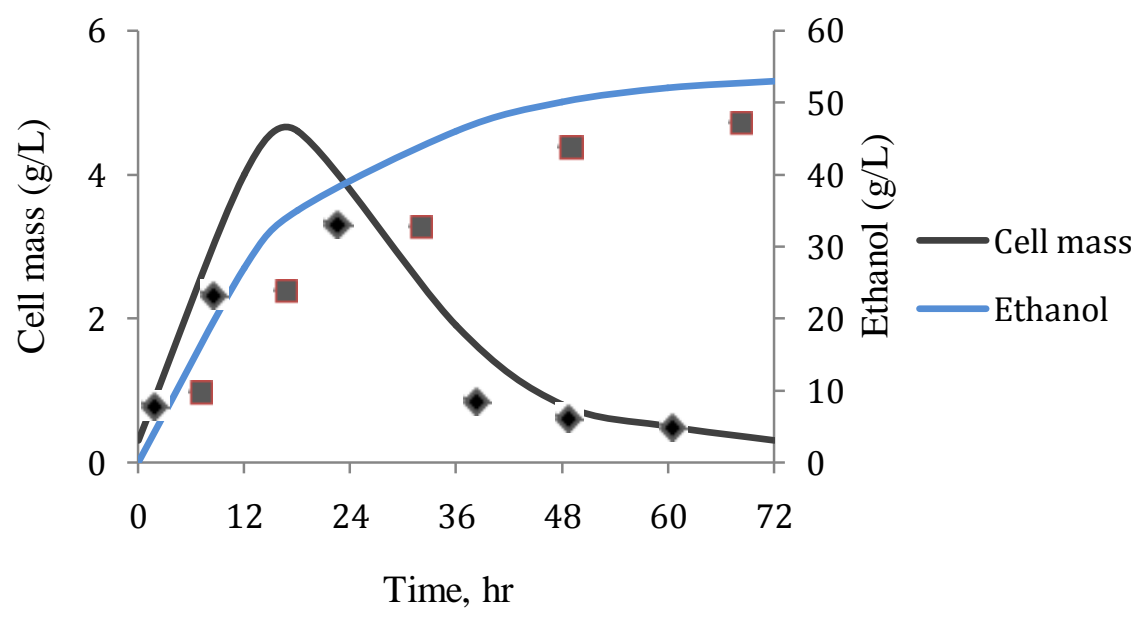

- Cell mass-exp—Cell mass-model ${ }^{\text {II }}$ Ethanol-exp—Ethanol-model

Figure 4: Experimental data and kinetic model prediction for SSO samples (substrate concentration at $60 \mathrm{~g} / \mathrm{L}$ ).

We believe that there are other reasons attributed to it for example, enzyme adsorption by lignin and/or obstruction of lignin on the surface of cellulose to the point that enzymes are not able to access cellulose (Collins, 2007).

Another set of experiments was conducted to assess the contribution of ethanol inhibition to the loss of cell viability. We expected good cell viability at lower SSO sample concentrations if inhibition was a major factor for cells lost. It was noticed in experiments that ethanol concentration was a major factor of declining cell 
viability, but at the same time, one or more factors other than ethanol inhibition that are not yet determined may contribute to the loss viability in SSCF using S. cerevisiae DA2416.

According to the sensitivity analysis conducted in this study a few parameters had the highest response values and exhibited the greatest influence on ethanol yields. Among them is a cellulose adsorption constant, enzymatic hydrolysis rate; ethanol yields from glucose and xylose; and ethanol tolerance. Table 3 presents the results of sensitivity analysis.

Table 3 Sensitivity analysis of kinetic parameters for SSO used in this work

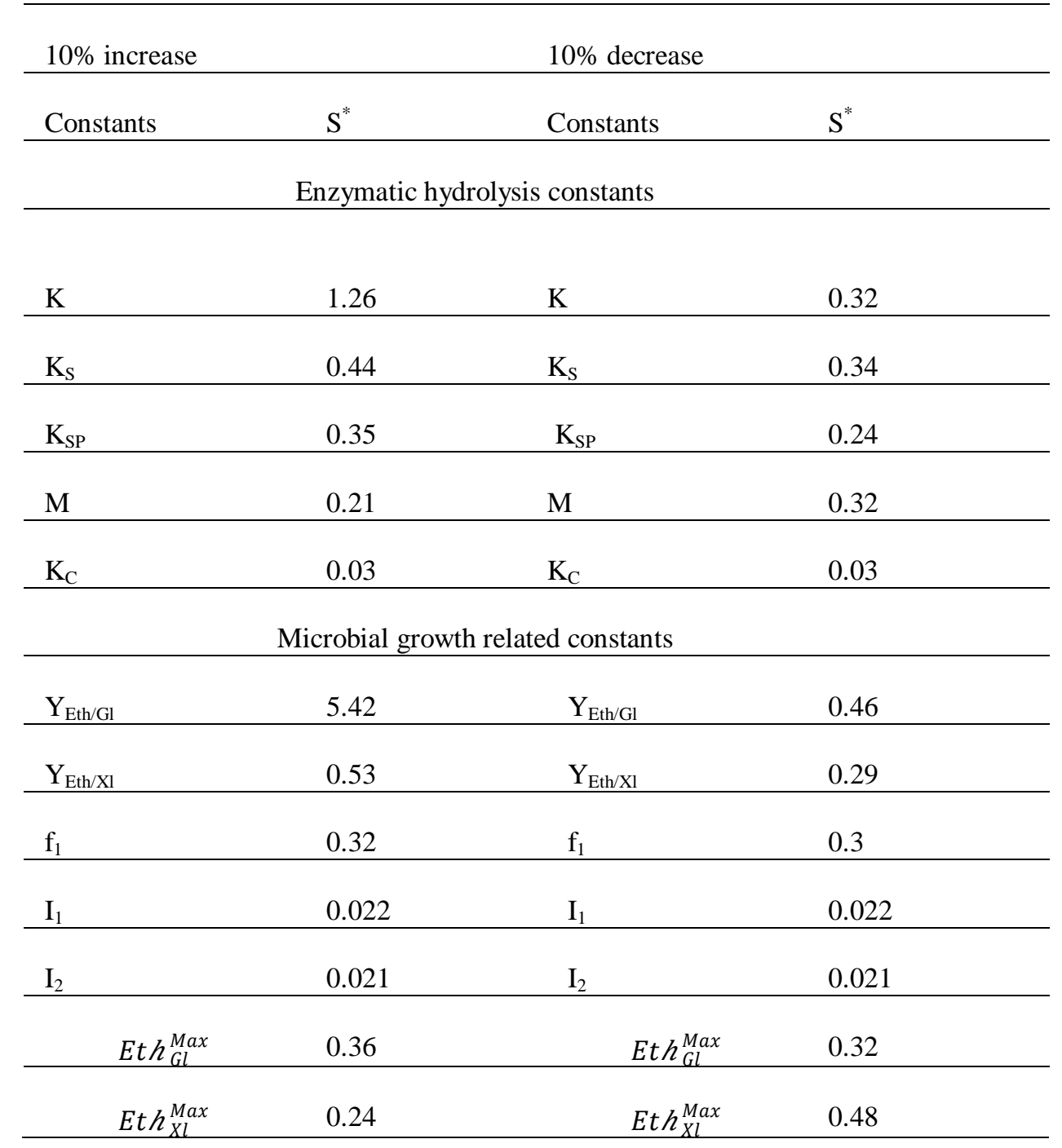

The sum of variance between the measured ethanol and the ethanol predicted by the model were calculated. Single parameters were varied to $\pm 10 \%$ of the experimental values and responses were calculated as absolute values based on the least square method (Bretscher, 1995). As seen from Table 3, cellulase adsorption constant $\mathrm{CS}$ had the highest response value followed by the cellulose enzymatic hydrolysis constant $\mathrm{k}$ among enzymatic hydrolysis constants. Evidently, a $10 \%$ decrease of CS is 5 times more sensitive than a $10 \%$ increase, demonstrating that increase in enzyme loading will be more effective than increase in substrate concentration for ethanol yield. 
Among microbial growth related constants, the ethanol yield from glucose and xylose exhibited the highest sensitivity, with ethanol tolerant related constants showing moderate sensitivity.

In summary, a comparison of performance of different $S$. cerevisiae strain parameters is presented in Table 4 below:

Table 4 Comparison performance of different S. cerevisiae strains

\begin{tabular}{llcc}
\hline & DA2416 & RWB222 $^{*}$ & D5A $^{*}$ \\
\hline g ethanol/g sugar consumed & 0.50 & 0.42 & 0.44 \\
\hline Final glucan conversion & 0.96 & 0.93 & 0.94 \\
\hline final xylan conversion & 0.94 & 0.95 & 0.93 \\
\hline ethanol yield, $\%$ & 114 & 105 & 100 \\
\hline
\end{tabular}

DA2416 - this work

RWB222* and D5A* - data from (Zhang and Lynd, 2010)

Experimental results demonstrated that $S$. cerevisiae DA2416 produced about $50 \mathrm{~g} / \mathrm{L}$ ethanol with an ethanol yield of $0.50 \mathrm{~g}$ of ethanol/g potential sugar fed for SSO in less than 5 days with $96 \%$ cellulose conversion. All strains exhibited almost the same value of glucan and xylan conversion. The total ethanol yield on sugar consumed was higher for DA2416 strains. As a result, we came to the conclusion that $S$. cerevisiae DA2416 was more tolerant to inhibitors than other two strains. Different substrates should be tested to validate the DA2416 strain in the future.

\section{Conclusions}

The SSO waste samples utilized in this research were pre-processed by the TSP and further used as substrates for all enzymatic hydrolysis and fermentation processes.

COSLIF pre-treatments were applied for cellulose extraction from processed SSO waste. Results indicated that a kinetic model with integrated values of experimentally defined SSO feedstock constants were able to predict ethanol yield accurately with diminutive variance from experiments. We examined that the cellulose adsorption constant, ethanol tolerance and ethanol yield played very important roles in the fermentation process. We also learned that a good fermenting strain should have the ability to withstand ethanol toxicity and common inhibitors. The discrepancy between experiments and model predictions, particularly at high substrate concentrations needs to be examined more comprehensively.

This study demonstrated and affirmed that $S$. cerevisiae DA2416 is a promising strain for SSO substrate in SSCF. In the future, the kinetic model used should be expanded to introduce the inference of lignin in lignocellulosic biomass.

\section{Acknowledgements}

The authors are greatly indebted to the technical support of the Department of Civil Engineering, and also the staff of graduate studies of Ryerson University for the facilities and assistance provided throughout this research. Special thanks are given to the Genencore Inc, a Denisco Division, Rochester, New York, USA, as well as Sigma Aldrich Corp., USA, for providing samples of Accellerase 1500, used in this study. Authors are 
grateful to Dr. Yong-Su Jin from Department of Food Science and Human Nutrition, University Illinois, USA for providing recombinant strain of S. cerevisiae DA2416 used in this study. The authors thank Dr. Khurram Shahzad Baig, from Chemical Engineering Department of Ryerson University for useful discussions. Optimum Waste \& Recycling Systems, Toronto, Canada, supplied biomass feedstock.

\section{References}

Bekmuradov, V., Luk, G., and Luong, R., 2014a, Improved cellulose and organic-solvents based lignocellulosic fractionation pre-treatment of organic waste for bioethanol production. American Journal of Engineering Research, 3(6), 177-185. Available from http://www.ajer.org/papers/v3\%286\%29/U036177185.pdf

Bekmuradov, V., Luk, G., and Luong, R., 2014b, Comparative ethanol productivities of two different recombinant fermenting strains on source separated organic waste. International Journal of Engineering Research and Applications, 4(10), 77-82. Available from http://www.ijera.com/pages/v4no10(v5).html

Bretscher O., 1995, Linear Algebra with Applications. 3rd edition, Upper Saddle River, (New Jersey: Prentice Hall).

Collins, K., 2007, The new world of biofuels: Implications for agriculture and energy". 2007. EIA Energy Outlook, Modeling, and Data Conference.

Department of Energy, USA, 1998, Annual Energy Review. Rep. DOE (IAE)-0384(97).

Dowe, N., and McMillan, J.D., 2008, SSF experimental protocols - lignocellulosic biomass hydrolysis and fermentation. Laboratory analytical procedure (LAP NREL/TP-510-42630). Technical report. National Renewable Energy Laboratory, Golden, Colorado, USA. Last accessed May 5, 2014 http://www.nrel.gov/docs/gen/fy08/42630.pdf

Ehsanipour, M., 2010). Acid pretreatment and fractionation of source separated organic waste for lignocellulosic saccharification. Master's thesis, Ryerson University, Civil Engineering Dept., Toronto, Canada.

Houghton, J., Weatherwax, S., and Ferrell, J., 2006, Breaking the biological barriers to cellulosic ethanol: A joint research agenda. DOE/SC- 0095.

Kim, T.H., and Lee, Y.Y., 2005, Pretreatment and fractionation of corn stover by ammonia recycle percolation process. Journal Bioresource Technology, 96, 2007-2013.

Kuyper, M., Toirkens, M.J., Diderich, J.A., Winkler, A.A., Dijken, J.P. van, and Pronk, J.T., 2005, Evolutionary engineering of mixed sugar utilization by xylose fermenting Saccharomyces serevisiae strain. FEMS Yeast Research, 10, 925-934.

Liu, H., Zhu, J.Y., and Chai, X.S., 2011, In situ, rapid, and temporally resolved measurements of cellulase adsorption onto lignocellulosic substrates by UV-Vis spectrophotometry, Langmuir, 27(1), 272-278.

Lynd, L.R., Weimar, P.J., Zyl, W.H. van, and Pretorius, I.S., 2002, Microbial cellulose utilization: fundamentals and biotechnology. Microbiology and Molecular Biology Reviews, 66(3), 506-677.

McMillan, J.D., Newman, M.M., Templeton, D.W., and Mohagheghi, A., 1999, Simultaneous saccharification and co-fermentation of dilute-acid pretreated yellow poplar hardwood to ethanol using xylose-fermenting Zymomonas mobilis. Journal Applied Biochemistry and Biotechnology, 77-79, 649-665. 
Valeriy Bekmuradov, Grace Luk, Michael Crupi, Elsayed Elbeshbishy / Kinetic Model Development on Source-Separated....

Mirzajani, M. (2009). The amenability of pre-treated source separated organic (SSO) waste for ethanol production. Master's thesis, Ryerson University, Civil Engineering Dept., Toronto, Canada.

Thermo Fisher Scientific Inc., 2011, Modified Lowry protein assay kit. Available from: http://www.thermoscientific.com/pierce

Mohageghi, A., Dowe, N., Schell, D., Chou, Y., Eddy, C., and Zhang, M., 2004, Performance of newly developed integrant of Zymomonas mobilis for ethanol production on corn stover hydrolysate. Biotechnology Letters, 26, 321-325.

Moxley, M., and Zhang, Y.-H.P., 2007, More accurate determination of acid-labile carbohydrate composition in lignocelluloses by modified quantitative saccharification. Journal Energy Fuels, 21, 3684-3688.

Optimum Waste\& Recycling Systems Ltd., 2010, ATS Technology Compost. Toronto, Ontario, Canada.

Ragauskas, A. J., Williams, C.K., Davison, B.H., Britovsek, G., Cairney, J., Eckert, C.A., Frederick, W. J., Hallett, J.P., and Liotta, C.L., 2006, The path forward for biofuels and biomaterials. Science, 311(5760), 484489.

Reifenberger, E., Boles, E., and Ciriacy, M., 1997, Kinetic characterization of individual hexose transporters of Saccharomyces serevisiae and their relation to the triggering mechanisms of glucose repression. European Journal of Biochemistry, 245(2), 324-333.

Ruiz, R., and Ehrman, T., 1996, Chemical analysis and testing laboratory analytical procedures. National Renewable Energy Laboratory (NREL). Determination of carbohydrates in biomass by high performance liquid chromatography. Lap-002.

Sedlak, M., and Ho, N.W-Y., 2004, Characterization of effectiveness of hexose transporters for transporting xylose during glucose and xylose co-fermentation by a recombinant Saccharomyces yeast. On-line Wiley Interscience (www.interscience.com). Microbiology and Virology(Yeast), 21, 671-684.

Shao, X., 2007, Cellulosic biomass to ethanol: kinetic modeling, scale up and reactor design. PhD thesis, Dartmouth College, Hanover, New Hampshire, USA.

South, S.R., Hogsett, D., and Lynd, L.R., 1995, Modeling simultaneous saccharification and fermentation of lignocellulose to ethanol in batch and continuous reactors. Journal Enzyme Microbial Technology, 17,797-803.

Stigler, S.M., 1986, The History of Statistics: The Measurement of Uncertainty Before. (Cambridge, MA: Belknap Press of Harvard University).

Teixeira, L.C., Linden, J.C., and Schroeder, H.A., 2000, Simultaneous saccharification and co-fermentation of peracetic acid-pretreated biomass. Journal of Applied Biochemistry and Biotechnology, 84-86, 111-128.

Uden, N. van (1989). Alcohol toxicity in yests and bacteria. CRC Press Inc. 1989. Boca Raton, Florida, USA.

Wang, Q.Q., Zhu, J.Y., Hunt, C.G., and Zhan, H.Y., 2012, Kinetics of adsorption, desorption, and re-adsorption of a commercial endoglucanase in lignocellulosic suspensions. Biotechnology and Bioengineering, 30(30), 124132.

Wyman, C.E., 1999, Biomass ethanol: Technical progress, opportunities and commercial challenges. Annual Review of Energy and the Environment, 24, 189-226. 
Xiao, Z., Zhang, X., Cregg, D.J., and Sadller, J.N., 2004, Effect of sugar inhibition on cellulases and $\beta$ glucosidase during enzymatic hydrolysis of softwood substrates. Journal Applied Biochemistry and Biotechnology, 115(1-3), 1115-1126.

Zhang, J., 2008, Simultaneous saccharification and co-fermentation of paper sludge. PhD thesis, Dartmouth College, Hanover, New Hampshire, USA.

Zhang, J., Shao, X., Townsend, O.V., and Lynd, L.R., 2009, Simultaneous saccharification and co-fermentation of paper sludge by Saccharomyces cerevisiae RWB222". Part 1: Kinetic modeling and parameters. Biotechnology and Bioengineering, 104(5), 920-931.

Zhang, J., and Lynd, L.R., 2010, Ethanol production from paper sludge by simultaneous saccharification and cofermentation using recombinant xylose fermenting microorganisms. Biotechnology and Bioengineering, 107(2), 235-244.

Zhang, Y.-H.P., Himmel, M., and Mielenz, J.R., 2006, Outlook for cellulase improvement: Screening and selection strategies. Journal Advanced Biotechnology, 24(5), 452-481.

Zhang, Y.-H. P., Ding, S.-Y., Mielenz, J.R., Elander, R., Laser, M., Himmel, M., McMillan, J.D., and Lynd, L.R., 2007, Fractionating recalcitrant lignocelluloses at modest reaction conditions. Biotechnology and Bioengineering, (97), 214-223.

Polymath Software, Willimantic, CT, USA, Retrieved from: http://polymath-software.com/ 